\title{
One-Dimensional Localization of Quantum Vortices in Disordered Josephson Junction Arrays
}

\author{
Alexander van Oudenaarden, S. J. K. Várdy, and J.E. Mooij \\ Department of Applied Physics and Delft Institute of Micro-electronics and Submicron-technology (DIMES), \\ Delft University of Technology, P.O. Box 5046, 2600 GA Delft, The Netherlands
}

(Received 26 August 1996)

\begin{abstract}
The influence of disorder on the transport of quantum vortices in one-dimensional arrays of Josephson junctions was studied experimentally. The vortices localize in disordered arrays for small vortex densities resulting in a vanishing transport at low temperatures, whereas, in a periodic array, transport remains possible. For large vortex densities the mobility of vortices is maintained in the disordered samples, due to the strong vortex-vortex interactions. [S0031-9007(96)01689-4]
\end{abstract}

PACS numbers: 74.50. $+\mathrm{r}$, 05.30.Jp, 72.15.Rn

Quantum mechanical particles in a periodic potential form energy bands; their wave functions extend through the system. When the periodicity is distorted the spatial extent of the wave functions is reduced. This phenomenon of Anderson localization [1] has been the subject of extensive studies during the past decades [2]. The lower the dimensionality of the system, the stronger the effect of disorder [3]. According to Mott and Twose [4], in onedimensional systems even weak disorder leads to strong localization. Experimentally, few systems are available for a controlled study of the dynamics of particles with one-dimensional motion. In this Letter we report on a study of the influence of disorder on the quantum transport of vortices in fabricated mesoscopic one-dimensional arrays of Josephson junctions. The great advantage of these systems is that disorder can be introduced in a very controlled way. We have compared the transport properties of samples with a slightly distorted periodicity of the potential to those of a periodic sample. We find that in the disordered systems the quantum transport is suppressed at low temperatures while vortex transport remains possible in the periodic system.

The motion of a vortex in a Josephson junction array is homologous to the motion of a particle in a potential landscape. The potential is periodic when all the junctions are identical. The energy barrier between two energy minima is proportional to the Josephson coupling energy $E_{J}=i_{c o} \Phi_{0} / 2 \pi$, where $\Phi_{0}=h / 2 e$ is the flux quantum and $i_{c o}$ the critical current of a single junction. Locally, this energy barrier can be tailored by varying $E_{J}$ of the junction. The mass of the vortex $m_{v}$ can be calculated quasistatically [5]: $m_{v}=\Phi_{0}^{2} C / 2 a^{2}$, here $C$ is the capacitance of the junction and $a$ the lattice constant. This quasistatic value can serve as an indication for the effective mass in the band. In a nearly free-vortex approximation, the bandwidth is close to twice the single electron charging energy $E_{C}=e^{2} / 2 C$. A vortex with a large mass in an array with high barriers $\left(E_{J} \gg E_{C}\right)$ behaves as a classical particle. However, for smaller mass and lower barriers $\left(E_{J} \approx E_{C}\right)$ the vortex exhibits quantum mechanical properties [5-7]. The vortices are bosonic particles with repulsive interaction. A previous Letter contains an experimental study of Mott localization of quantum vortices in periodic samples [7]. When the vortex density is commensurate with the one-dimensional lattice potential, the vortices localize due to the effects of vortex-vortex interaction. In this Letter we report on an experimental study of the influence of disorder on the quantum transport of vortices.

The samples consist of a square network of Josephson junctions in which each superconducting island is coupled to four nearest neighbors (square cell). The length $L$ of the array is very large $(L=1000$ cells $)$ compared with the width $W(W=7$ cells). Along the length of the array the islands at both edges are connected by a narrow superconducting strip (busbar). The vortices are repelled by these busbars and are therefore confined to one dimension. The bias current, which exerts a force on the vortex in the length direction, is injected in the middle of the busbar. The voltage, which is induced when the vortices move, is probed at one end of the busbars [Fig. 1(a)]. To study the influence of disorder, a periodic superlattice was constructed and compared with two slightly disordered

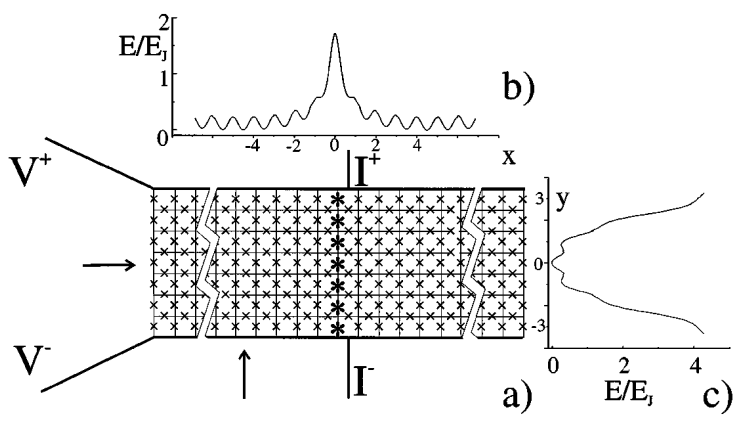

FIG. 1. (a) Sketch of the sample layout. The resistance is determined by a four terminal measurement. The bias current is applied in the middle of the sample and the voltage is probed at one end. The lattice junctions and the barrier junctions are denoted by crosses and stars, respectively. (b) The potential landscape for a vortex traveling through the middle path (along horizontal arrow) near a barrier. (c) The potential landscape perpendicular to the length direction (along vertical arrow). 
superlattices. A barrier was created by enlarging $E_{J}$ of the junctions at the barrier position with a factor of two. These junctions will be called "barrier junctions," whereas the other junctions will be called "lattice junctions." In Fig. 1(a) it is illustrated how a barrier is introduced in the array. A column of junctions with Josephson coupling energy $E_{J}$ is replaced by junctions with Josephson coupling energy $2 E_{J}$. To estimate the actual static potential landscape numerical simulations were performed. The total potential energy $E=\sum_{i} E_{J i}\left(1-\cos \phi_{i}\right)$ was computed iteratively by solving Kirchhoff's law at each island. $E_{J i}$ is the Josephson coupling energy of junction $i$ and $\phi_{i}$ the gauge invariant phase difference of junction $i$. In the calculation induced magnetic fields were neglected, which is reasonable because the geometrical selfinductance $L_{s}=\mu_{0} a$ is much smaller than the Josephson inductance $L_{J}=\Phi_{0} /\left(2 \pi i_{c o}\right)$. A more elaborate calculation for a large two-dimensional array, taking into account all inductance effects, was done by Phillips et al. [8]. The position of the vortex is defined by fixing the phases of four islands. The remaining phases are computed. The phases of the islands which are connected by a busbar are equal. In Fig. 1(b) the result of the calculation is shown. Far from the barrier, the cell-to-cell barrier is $0.2 E_{J}$, which corresponds to the results of Lobb et al. [9] for a uniform two-dimensional array. The barrier junction causes an energy barrier of $1.7 E_{J}$. The perturbation of the potential spreads out over approximately 3 cells on both sides. In Fig. 1(c) the potential energy perpendicular to the length axis is shown. The vortices travel along the middle path $(y=0)$ because of the repelling busbars.

A comparison was made between periodic and disordered samples. In the periodic superlattice, the distance between two barriers is always $10 a(\delta=0)$. In others disorder was introduced using a uniform distribution. In the least disordered sample $(\delta=1)$ the barriers are separated by $9 a, 10 a$, or $11 a$ with equal probabilities. In the other disordered sample $(\delta=2)$ the barriers are separated by $8 a, 9 a, 10 a, 11 a$, or $12 a$ also with equal probabilities. Each sample contains 100 barriers.

Samples were fabricated using electron beam lithography and a shadow evaporation technique. The junctions are small $\mathrm{Al}-\mathrm{Al}_{2} \mathrm{O}_{3}-\mathrm{Al}$ tunnel junctions. The capacitance $C$ of the lattice junctions is estimated to be $1 \mathrm{fF}$. The barrier junctions have a capacitance of about $2 \mathrm{fF}$. The normal state array resistance $R_{n}$ is $(91 \pm 1) \Omega$ for all three samples. Assuming that the normal state resistance of the barrier junctions is half the resistance of the lattice junctions, the normal state lattice junction resistance $r_{n}$ equals $(14.3 \pm 0.2) \mathrm{k} \Omega$. The Ambegaokar-Baratoff result for aluminum $\left(i_{c o} r_{n}=300 \mu \mathrm{V}\right)$ leads to $E_{J} / k_{B} \approx 0.5 \mathrm{~K}$ and $E_{J} / E_{C} \approx 0.52$. The lattice constant $a$ is $1.2 \mu \mathrm{m}$ and the cell area $S$ is $2 \mu \mathrm{m}^{2}$. The quasistatic vortex mass is approximately $10^{-3}$ times the electron mass.

Experiments were performed in a dilution refrigerator. The electrical signals were filtered by means of $R C$ and microwave filters at the temperature of the mixing chamber. At room temperature the leads were additionally filtered by $\Pi$ filters. The sample was screened from magnetic inference by placing it in a superconducting shield inside a $\mu$-metal box. A small magnetic field was applied by two superconducting coils in the Helmholtz configuration. The one-dimensional frustration $n$ is defined as the magnetic flux $W B S$ divided by $\Phi_{0}$. For an infinite system, $n$ equals the one-dimensional vortex density.

To characterize the quality of the samples, the depinning current $I_{d}$ as a function of $n$ was measured. The depinning current is defined as the current at which the voltage over the array exceeds $2 \mu \mathrm{V}$. At the depinning current the vortices have just enough energy to overcome the barrier potentials. Below $I_{d}$ vortex transport is only possible by thermal activation over the barriers or quantum transport through the energy band. In Fig. 2(a) the measured $I_{d}$ is plotted as a function of $n$ for the three samples. For the periodic superlattice $(\delta=0)$, distinct peaks are observed at $n=p / 10$, where $p$ is an integer number. These peaks reflect the periodic arrangement of the barriers, which are spaced by 10 lattice cells. When disorder is introduced, most peaks disappear. For $\delta=1$, two peaks are observed on both sides of the " $n=0$ " peak, whereas for $\delta=2$ only one peak on both sides is observed. For $\delta=1$ and $\delta=2$, small peaks around $n=1$ and $n=2$ are observed. These peaks are due to the periodicity of the lattice junctions. To obtain a more quantitative comparison the Fourier transformation of the energy landscape was computed. The potential of the barrier is approximated by the potential depicted in Fig. 1(b). More than 4 cells from the barrier

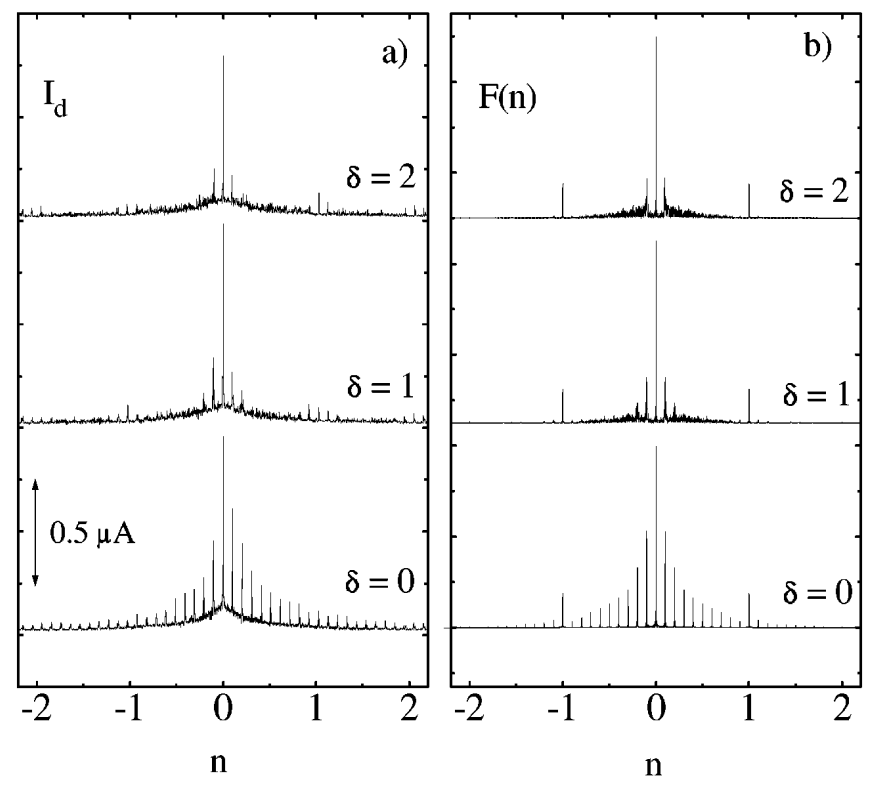

FIG. 2. (a) The experimental depinning current $I_{d}$ as a function of $n$ at $T=30 \mathrm{mK}$. (b) Calculated Fourier spectra $F(n)$ of the potential landscape of the three samples. The units on the $y$ axis are arbitrary. 
the potential is assumed to be sinusoidal. In Fig. 2(b) the calculated Fourier spectra of the potential landscapes of the three samples are shown. A close resemblance between the calculated spectra and the measurements is observed, which reflects the high quality of the samples.

To determine the quantum transport properties of the vortices the zero-bias resistance $R_{0}$ was measured by means of a lock-in technique. The resistance was measured with a maximum bias current of $2 \mathrm{pA}$ per junction. For these small currents the resistance was determined in the linear response regime. The voltage resolution is $0.4 \mathrm{nV}$. For the periodic sample, $R_{0}$ drops sharply to zero at commensurate densities $n=p / 10$. This localization is due to the effects of the repulsive vortex-vortex interaction and is similar to the forming of a Mott insulator which was studied in detail in Ref. [7]. We did not observe the forming of a Mott insulator at $n=1 / 3$ and $n=2 / 3$ as was previously observed [7]. Below, we are explicitly interested in the effect of disorder on the quantum transport at noncommensurate densities. In Fig. 3 the logarithm of $R_{0}$ normalized to the normal state array resistance $R_{n}$ is plotted as a function of $E_{J} / k_{B} T$ at a one-dimensional vortex density $n=0.44$. Here $E_{J}$ is the Josephson coupling energy of the lattice junctions. At high $T\left(E_{J} / k_{B} T<0.8\right)$ no significant difference was observed between the different samples. The vortex transport is thermally activated. The corresponding energy barrier for the thermally activated transport is approximately $3 E_{J}$ for all three samples. This barrier is larger than the computed value $1.7 E_{J}$. This discrepancy is not yet understood. When the temperature is lowered below $600 \mathrm{mK}\left(E_{J} / k_{B} T>0.8\right)$, a significant difference is observed between the periodic sample $(\delta=0)$ and the two

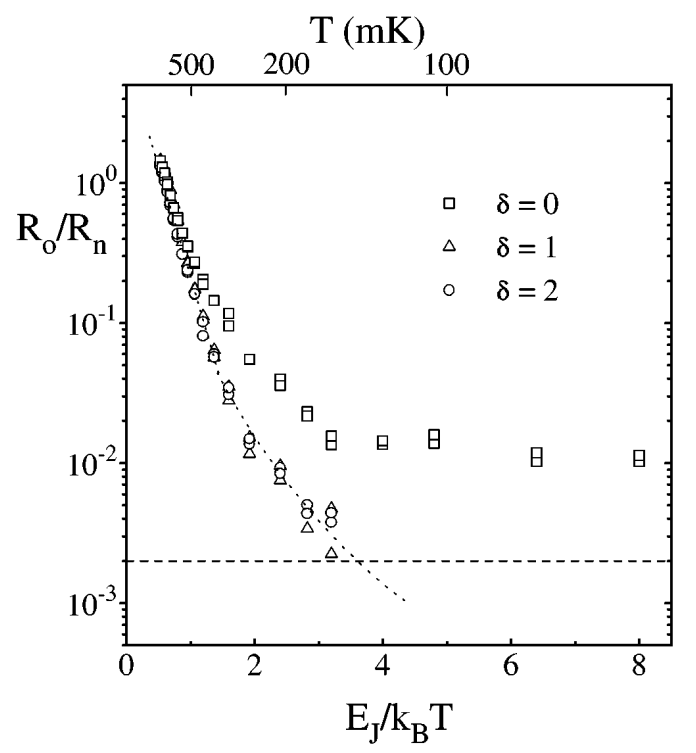

FIG. 3. The logarithm of the normalized resistance $R_{0} / R_{n}$ as a function of the normalized reciprocal temperature $E_{J} / k_{B} T$ for $n=0.44$. The noise floor at $R_{0} / R_{n}=2 \times 10^{-3}$ is denoted by the dashed line. The dotted line serves as a guide for the eye. disordered samples. For the periodic array a finite $R_{0}$ $\left(R_{0} / R_{n}=0.015\right)$ is observed at the lowest temperatures. In contrast, for the disordered arrays the zero-bias resistance disappears below our experimental resolution. In other words, transport is possible in the periodic sample resulting in a finite zero-bias mobility of the vortices, whereas in the disordered arrays the vortices are localized $\left(R_{0}=0\right)$. Between the two disordered samples no clear difference is observed. This observation is consistent with the fact that in a one-dimensional system even very weak disorder causes localization. The localization length in the disordered samples is of the order of the distance between two barriers $(\approx 10$ cells $)$.

For $n<0.3$ the zero-bias resistance is too small to resolve even for the periodic sample. For $0.3<n<0.8$ the zero-bias resistance of the periodic sample is significantly larger than the zero-bias resistance of the disordered samples. However, around $n=1$ the transport properties of the three samples are not significantly different. In Fig. 4 the logarithm of the normalized resistance is shown as a function of the reciprocal temperature for a large vortex density $(n=1.08)$. Whereas for low densities a clear difference is observed between ordered and disordered samples, at high densities no significant difference in transport properties is measured. At high densities the distance between two neighboring vortices is small, and therefore the repulsive vortex-vortex interaction becomes more important than at low densities. The experimental fact that transport is possible in the disordered lattices for $n \approx 1$ indicates delocalization of the vortices due to the repulsive interaction.

To summarize, we have studied the transport of quantum vortices in very long and narrow arrays of Josephson

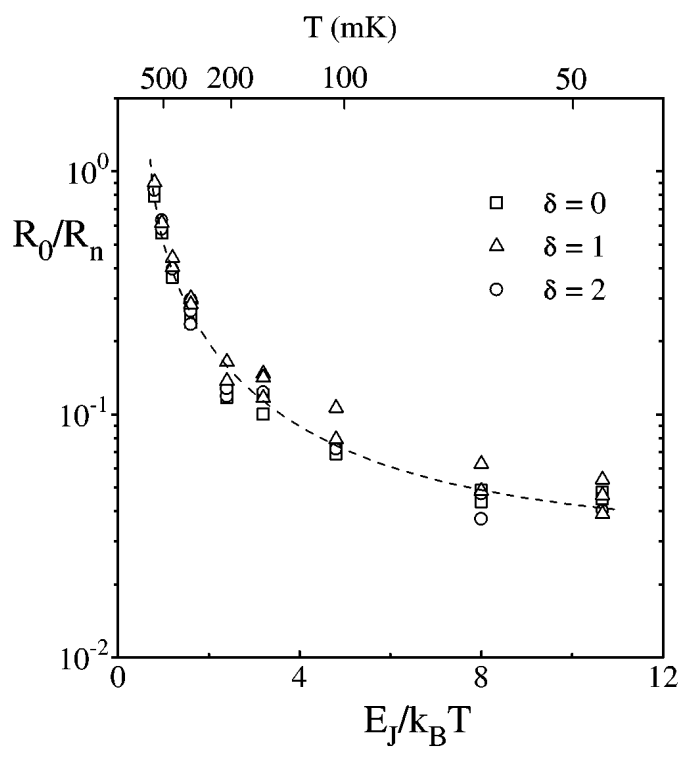

FIG. 4. The logarithm of the normalized resistance $R_{0} / R_{n}$ as a function of the normalized reciprocal temperature $E_{J} / k_{B} T$ for $n=1.08$. The dashed line is a guide for the eye. 
junctions, with controlled presence and absence of disorder. At high temperatures no difference was observed between ordered and disordered samples; transport is governed by thermal activation. At low temperatures for low vortex densities, localization was clearly observed in the disordered samples. For larger densities the vortices delocalize in the disordered systems. This indicates the important role of vortex-vortex interaction.

The idea of one-dimensional vortex localization grew from discussions of W. J. Elion and A. Stern. We want to thank M. P. M. Robbens and S. L. Yeung for their help with the simulations. The Delft Institute of Micro-electronics and Submicron-technology (DIMES) is acknowledged for the support in fabrication. The work was supported by the Dutch Foundation for Fundamental Research on Matter (FOM).

[1] P. W. Anderson, Phys. Rev. 109, 1492 (1958).
[2] P. A. Lee and T. V. Ramakhrisnan, Rev. Mod. Phys. 57, 287 (1985); B. Kramer and A. MacKinnon, Rep. Prog. Phys. 56, 1469 (1993).

[3] E. Abrahams, P.W. Anderson, D. C. Licciardello, and T. V. Ramakrishnan, Phys. Rev. Lett. 42, 673 (1979).

[4] N. F. Mott and W. D. Twose, Adv. Phys. 10, 107 (1961).

[5] E. Simanek, Solid State Commun. 48, 1023 (1983); S. E. Korshunov, Physica (Amsterdam) 152B, 261 (1988); A. I. Larkin, Yu. N. Ovchinikov, and A. Schmid, Physica (Amsterdam) 152B, 266 (1988); U. Eckern and A. Schmid, Phys. Rev. B 39, 6441 (1989).

[6] H. S. J. van der Zant, F. C. Fritschy, W. J. Elion, and J.E. Mooij, Phys. Rev. Lett. 69, 2971 (1992); W. J. Elion, J. J. Wachters, L. L. Sohn, and J. E. Mooij, Phys. Rev. Lett. 71, 2311 (1993).

[7] A. van Oudenaarden and J.E. Mooij, Phys. Rev. Lett. 76, 4947 (1996).

[8] J. R. Phillips, H.S. J. van der Zant, J. White, and T.P. Orlando, Phys. Rev. B 47, 5219 (1993).

[9] C. J. Lobb, D. W. Abraham, and M. Tinkham, Phys. Rev. B 27, 150 (1983). 